\title{
Thermal Modeling of an Implantable Brain Focal Cooling Device
}

\author{
H. Dinis*, J. Fernandes*, V. Silva, I. Colmiais, P. M. Mendes
}

\begin{abstract}
An implantable focal cooling device based on a Peltier is proposed in this paper. The building blocks of this device, namely its battery management, wireless power transfer, communication, and power management modules are presented and discussed. Simulated and experimental data in a deionized water phantom at $37^{\circ} \mathrm{C}$ validate the use of the Peltier as a focal cooling device, and simulations in rat brain further confirm this conclusion.
\end{abstract}

\section{INTRODUCTION}

According to Fisher et al. [1], epilepsy is a disease that is characterized by the occurrence of two unprovoked seizures that are separated by a time frame of 24 hours or one seizure associated with other factors that imply a high probability of seizure occurrence. This condition is also characterized by its seizures' unpredictability, while provoking other health problems. It's the fourth most common neurological disease and it affects people of all ages. Currently, it affects around 65 million people, of which $70 \%$ are prescribed anti-epileptic drugs (AEDs). The remaining 30\%, however, must rely on other therapies, such as vagus nerve stimulation (VNS), deepbrain stimulation (DBS) or surgery to remove the epileptogenic region of the brain [2]. Focal brain cooling has been shown to be another effective therapy for intractable epilepsy [3].

In this paper, a fully implantable device for focal brain cooling in rats is proposed. The objective of controlling erroneous neuronal activity will be achieved by a Peltier module which, by means of an electrical current flowing through its terminals, can create a temperature differential between its two sides. The cold side will be used to cool down the epileptic focus on the brain to prevent inherent complications, while the hot side will be kept below $43{ }^{\circ} \mathrm{C}$ to prevent irreversible neuronal damage [4]. The temperatures achieved by the Peltier were evaluated using a simulation in water at $37{ }^{\circ} \mathrm{C}$ and validated experimentally. Another simulation in a rat's brain was also made, while experimental procedures are currently being performed.

Over the next sections of this paper, the components of the proposed implantable device will be unveiled and described.

\section{Proposed Implantable Device}

The device developed by Fujii et al. [5] is regarded as the state of the art in implantable focal brain cooling devices. It is based on a Peltier device, $4 \mathrm{~mm}$ in width and length and $2 \mathrm{~mm}$ in thickness. An aluminum heat sink with an internal circulating water channel was attached to the Peltier's hot side in order to help dissipate the generated heat. Their device proved capable of cooling rat brain to the point of suppressing electric discharges (ED), therefore demonstrating that it is a feasible application. Nevertheless, the proposed device is not fully implantable, which is a crippling drawback for its clinical application.

Our device proposal aims to improve upon the existent one by means of eliminating the need to use the water circulation system. To achieve that, another temperature management mechanism that leads to size and weight reduction is expected, as well as the complete removal of the external water pumps and pipes. Furthermore, wireless operation (communication and power) is desirable in order to have a fully automatic and independent system that can be chronically implanted in rats without limiting their natural mobility.

\section{A. Device concept}

The device is being designed for placement on a rat's brain, therefore it has to be small, protect the inner parts of the Peltier from fluids and provide enough heatsink capabilities to reduce the temperature on the hot side. The Peltier module was attached to a small aluminum heatsink $\left(10 \times 15 \mathrm{~mm}^{2}\right)$ using thermal heatsink paste. With the use of CAD tools and a 3D printer, a small shell was built for the Peltier module and its heatsink, promoting a waterproofing of the device, as seen in Fig. 1. When using this system in an aqueous mean, this impermeability will ensure that the system continues to function, as the presence of an electrically conductive substance between the pillars of the Peltier will change its resistance and, consequently, its behavior, making it less capable of achieving large temperature differences between its two sides. When placing the implant in a rat head, this could be a problem because some blood or even cerebrospinal fluid can infiltrate inside the Peltier and change the cooling capacity of the system. The full device measures $10 \times 15 \times$ $5 \mathrm{~mm}^{3}$ and, because of the easily customizable 3D printed shell, it can be adapted according to the surgeon's requests.

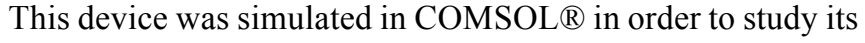
effectiveness in cooling brain cells.

\section{B. Wireless Power Transfer}

In order to power the Peltier device over an amount of time

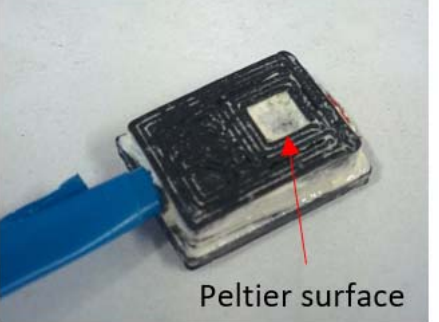

Figure 1 - Photograph of the concept device for brain cooling. 
comparable to those of other implantable devices, such as pacemakers, a large battery would be required. Considering that the Peltier requires $400 \mathrm{~mW}$ during 30 seconds, a $3.33 \mathrm{mWh}$ battery would be required for a single cooling action. For the sake of the example, lets suppose that the device would need to be activated 5 times every day, and that our target was a 5-year battery lifetime. Considering only the Peltier's power requirements, the implant would need a $30 \mathrm{Wh}$ battery, the equivalent to 3-5 smartphone batteries. Such a large battery is, of course, incompatible with implantable devices, especially in the human head. In order to reduce its size, power has to be supplied to the device resorting to another method, which is where wireless power transfer (WPT) comes in. While the battery is not being used to power the Peltier, it could be recharged from the outside using an electromagnetic wave. Its required capacity could, consequently, be drastically reduced, potentially to $3.33 \mathrm{mWh}$, the energy requirement of a single Peltier $30 \mathrm{~s}$ operation.

For the device to be WPT ready, it needs to have an integrated antenna to transform the electromagnetic wave into an electric current, and an RF-DC converter. These are responsible for converting the high-frequency signal into a DC supply, which will then recharge the battery. The antenna must be small enough not to significantly add to the device's final volume and weight. To meet these criteria, the heatsink, a metallic sheet, has been proposed to be used as an antenna as well [6].

Finally, when designing WPT links, power levels must be carefully chosen, as the IEEE imposes a limit to the amount of radiation that a biological tissue can be exposed to, in the form of the specific absorption rate (SAR) value. The design of a WPT link suitable for this application may not be possible resorting to a single transmission antenna, as high power levels are demanded, and a dual antenna setup has already been reported in [7], where promising results were achieved.

\section{Control electronics}

The chip containing all control, communication and power management circuits is being designed using $0.18 \mu \mathrm{m}$ CMOS technology. Fig. 2 contains a block diagram of the chip illustrating the aforementioned circuits, which will be explained and analyzed in the following sections of this paper.

\section{1) $R F-D C$ converter}

This block is responsible to convert an RF wave into a DC signal. Since the human body is a lossy RF wave propagation medium, due to the tissues' conductivity, the efficiency of the antenna and the RF-DC converter must be as high as possible.

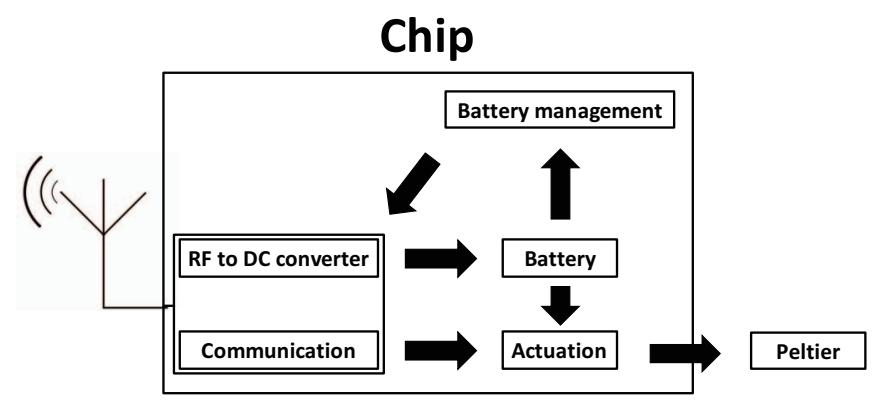

Figure 2 - Block diagram of the chip.

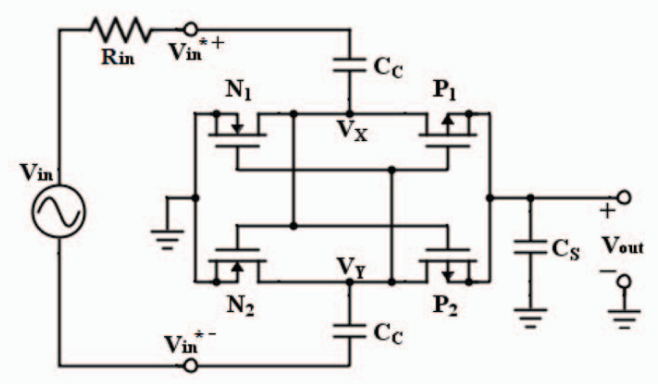

Figure 3 - Schematic of the base element of the RF-DC converter [8].

In order to achieve this, a diode-connected MOSFET was selected as the rectifying element.

A threshold voltage cancellation technique was employed with the objective of improving the efficiency of conversion. The base element of the RF-DC converter is represented in Fig. 3. As it is necessary to maximize the power conversion efficiency, multiple base elements are combined in a cascade arrangement.

The architecture of the rectifier allows an antenna to be connected directly to its input, while a battery charging circuit was designed and connected to its output. This charger circuit was described in [9].

\section{2) Communication}

The device is being designed with the ultimate goal of being implanted in the brain, therefore several device parameters must be monitored from the outside world in order to ensure a correct operation of the implant, e.g., the battery charging status and the Peltier's status (on or off). Additionally, the Peltier has to be controlled from the outside, hence the need for the control electronics to receive and understand commands such as turning on/off the Peltier and controlling its input current. The base of the communication block is a low noise amplifier (LNA). There are many types of LNAs, but they can generally be fitted into two main categories: with and without inductors. As die area is a design constraint and should be minimized, the chosen LNA is an inductorless type. Several logic circuitries will be implemented with the purpose of sending/receiving the aforementioned signals.

\section{3) Battery management}

For proper system operation, battery management is a necessity, as it is imperative to guarantee that the communication block only turns on when the battery is charged and turns off when the battery is discharged. In other words, the communication block must be off to guarantee that the power received by the antenna is fully converted into a DC supply that charges the battery. An operation example of that block is illustrated in Fig. 4. As it can be seen, when the battery is charging, the communication block is off. It is turned on when the battery is charged above $2.9 \mathrm{~V}$ and, finally, when the battery depletes to below $1.9 \mathrm{~V}$, the communication block is turned off again, as it can not properly function with voltages lower than $1.9 \mathrm{~V}$, simultaneously allowing for the battery to be recharged again. 


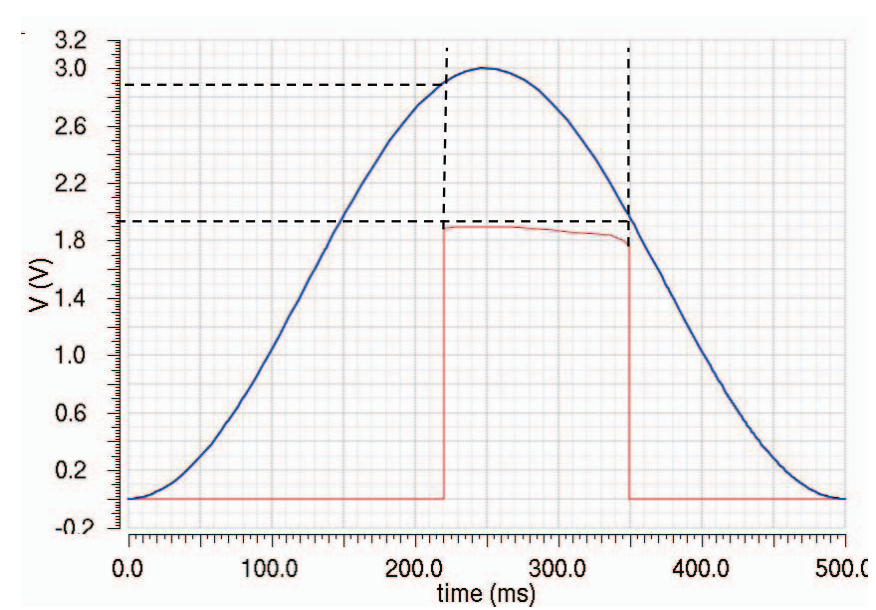

Figure 4 - Operation example of the battery management circuitry. Blue: battery voltage. Red: communication block's on/off signal.

\section{4) Actuation}

As previously described, the main objective of this project is cooling down a section of the brain. Such can only be achieved with the main component of the implant, the Peltier module. The chip will include current sources of different values connected to the Peltier so that, resorting to the communication block, it is possible to control the power that is injected to the device (and consequently its cooling potential) from the outside.

\section{RESUltS AND DISCUSSION}

To determine whether or not the selected Peltier device can be used to cool down brain cells, some simulations were performed. With such simulations, the device's operation conditions can be easily changed to understand what can be modified and adapted in order to effectively cool down brain cells. This thermoelectric simulation also allows the study of the necessary currents to power the Peltier with, as well as the depths and volumes of cooled tissue that can be achieved with this device. In Fig. 5 it is possible to observe the simulated results of the Peltier inside water.

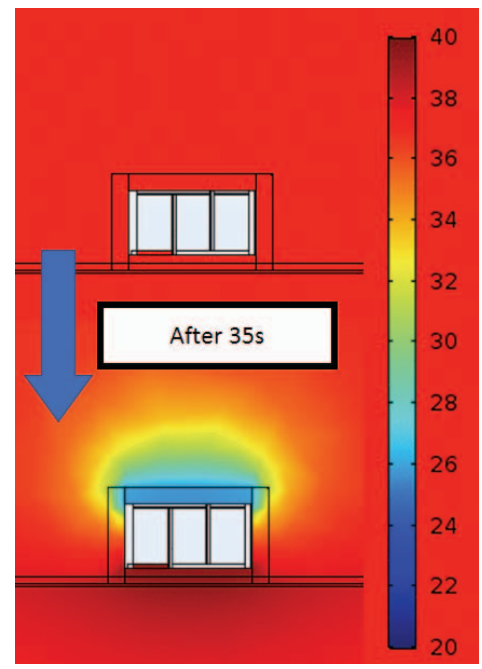

Figure 5 - Simulation results of the device in aqueous mean.

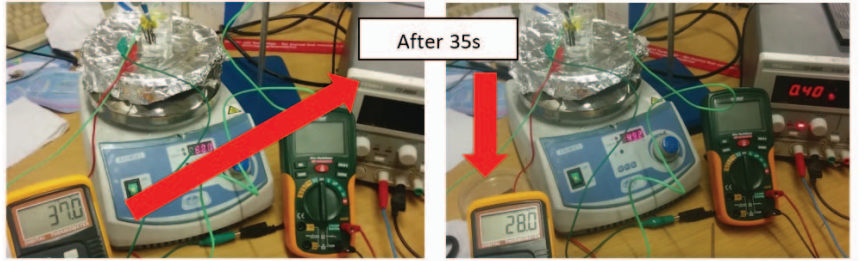

Figure 6 - Experimental setup for testing in $37{ }^{\circ} \mathrm{C}$ water.

The first phantom used to test the device was deionized water at $37{ }^{\circ} \mathrm{C}$, the normal temperature of the human brain, as illustrated in Fig. 6. With this phantom, the minimum brain temperature that this device can produce can be estimated.

Comparing the simulated with the measured results (Table 1) it is possible to verify that the values are very similar. These results led us to conclude that the model can be used for future simulations and to simulate the behavior of the Peltier device when in contact with brain cells.

When simulating the Peltier device with a model of a rat brain, it can be seen that this device is capable of cooling down a large volume of brain cells. In Fig. 7, it is observable that the Peltier module can cool down a significant volume of brain tissue to temperatures bellow $30^{\circ} \mathrm{C}$ (the necessary temperature to stop the electrical activity of rat brain cells).

TABLE I. COMPARISON BETWEEN SIMULATED AND MEASURED RESULTS

\begin{tabular}{|c|c|c|}
\hline Time $(\mathrm{s})$ & Simulated $\left({ }^{\circ} \mathrm{C}\right)$ & Measured $\left({ }^{\circ} \mathrm{C}\right)$ \\
\hline 0 & 35,88 & 37 \\
\hline 5 & 33,87 & 35,8 \\
\hline 10 & 30,75 & 28,7 \\
\hline 15 & 29,46 & 28,2 \\
\hline 20 & 28,76 & 28,2 \\
\hline 25 & 28,31 & 28,0 \\
\hline 30 & 28,03 & 27,9 \\
\hline 35 & 27,78 & 28,0 \\
\hline 40 & 27,63 & 28,0 \\
\hline 45 & 27,53 & 28,1 \\
\hline
\end{tabular}

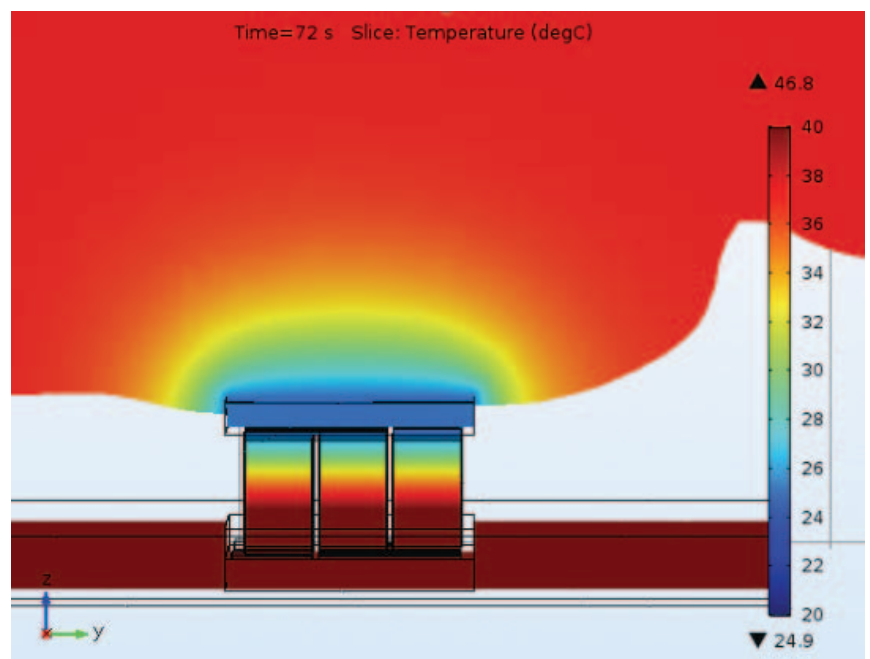

Figure 7 - Peltier device in contact with brain cells. 


\section{CONCLUSION}

An implantable focal brain cooling device for epilepsy treatment which aims to improve upon the current state of the art has been presented. The device's building blocks, such as control electronics and WPT module, were described. Thermal simulations of the Peltier module were performed and validated with experimental results. They indicated that the device was capable of cooling down to $28{ }^{\circ} \mathrm{C}$ when operating inside $37{ }^{\circ} \mathrm{C}$ water. The next step was simulating the Peltier in a rat brain. It was concluded that a large brain area was cooled, thus allowing for ED suppression. As short term future work, the device will be further improved upon, namely its shell, and will be tested in-vivo.

\section{ACKNOWLEDGMENT}

Work supported by FCT under project PTDC/EEITEL/5250/2014 - POCI-01-145-FEDER-16695, by FEDER funds through Projeto 3599 - Promover a Produção Científica e Desenvolvimento Tecnológico e a Constituição de Redes Temáticas (3599- PPCDT), and under grant SFRH/BD/100649/2014.

\section{REFERENCES}

[1] R. S. Fisher et al., "ILAE Official Report: A practical clinical definition of epilepsy," Epilepsia, vol. 55, no. 4, pp. 475-482, Apr. 2014.

[2] NHS, "Epilepsy - Treatment," 2014. [Online]. Available: http://www.nhs.uk/Conditions/Epilepsy/Pages/Treatment.aspx. [Accessed: February 2017]

[3] R. Fisher, "Therapeutic Devices for Epilepsy," Ann. Neurol., vol. 71, no. 2, pp. 157-168, 2012.

[4] J. Fernandes, H. Dinis, L. M. Gonçalves, P. M. Mendes, "Implantable microdevice with integrated wireless power transfer for thermal neuromodulation applications," 18th International Conference on Ehealth Networking, Application \& Services (Healthcom), Munich, Germany, 2016.

[5] M. Fujii, H. Fujioka, et al, "Application of Focal Cerebral Cooling for the Treatment of Intractable Epilepsy", Neurol Med Chir (Tokyo) 50, p839-844, 2010.

[6] H. Dinis, J. Fernandes, P. M. Mendes, "Slot Antenna Design for a Wirelessly Powered Implantable Microcooler for Neuronal Applications", 11th European Conference on Antennas and Propagation, 2017, Accepted.

[7] H. Dinis, I. Colmiais, P. M. Mendes, "A Multiantenna Approach to Maximize Wireless Power Tranfered to Implantable Devices", 2017 International Applied Computational Electromagnetics Society (ACES) Symposium, 2017, Accepted.

[8] K. Kotani, A. Sasaki, and T. Ito, "High-efficiency differential-drive CMOS rectifier for UHF RFIDs," IEEE J. Solid-State Circuits, vol. 44, no. 11, pp. 3011-3018, 2009.

[9] B. Do Valle, C. T. Wentz, and R. Sarpeshkar, "An area and powerefficient analog li-ion battery charger circuit," IEEE Trans. Biomed. Circuits Syst., vol. 5, no. 2, pp. 131-137, 2011. 